\title{
Molecular cloning of porcine growth differentiation factor 9 (GDF-9) cDNA and its role in early folliculogenesis: direct ovarian injection of GDF-9 gene fragments promotes early folliculogenesis
}

Takashi Shimizu, Yasunori Miyahayashi, Masaki Yokoo, Yumi Hoshino, Hiroshi Sasada and Eimei Sato

Laboratory of Animal Reproduction, Graduate School of Agricultural Science, Tohoku University, 1-1 Tsutsumidori-amamiyamachi, Aoba-ku, Sendai 981-8555, Japan

Correspondence should be addressed to T Shimizu, Department of Pathology, Institute of Development, Aging and Cancer, Tohoku University, 4-1 Seiryo-machi, Aoba-ku, Sendai 980-8575, Japan; Email: shimizut@idac.tohoku.ac.jp

\begin{abstract}
Growth differentiation factor-9 (GDF-9) is a growth factor secreted by oocytes in growing ovarian follicles. To investigate the ovarian function of GDF-9 in pigs, we first cloned porcine GDF-9 complementary DNA (cDNA), and then injected its gene fragments into the ovary in gilts. Porcine GDF-9 has open reading frame (ORF) homologies of 81.4\%, 84.6\%, 84.2\%, 72.7\% and $72.6 \%$ with its human, bovine, ovine, rat and mouse counterparts respectively. Regarding the deduced amino-acid sequence of the mature protein, the corresponding homologies reach $92.1 \%, 97.8 \%, 97.0 \%, 89.6 \%$ and $88.1 \%$ respectively. To investigate the role of GDF-9 in early folliculogenesis, the ovaries of 2-month-old prepubertal gilts were injected with GDF-9 gene fragments. The injection of porcine GDF-9 gene fragments resulted in an increase in the number of primary, secondary and tertiary follicles, concomitant with a decrease in the number of primordial follicles. These results indicated that exogenous GDF-9 can promote early folliculogenesis in the porcine ovary, and that a technique for direct ovarian injection of GFD-9 gene fragments may contribute to a novel therapy for prevention and treatment of infertility associated with ovarian dysfunction.

Reproduction (2004) 128 537-543
\end{abstract}

\section{Introduction}

The development of ovarian follicles is a complex process dependent upon endocrine regulation involving hypophyseal gonadotropins, and regulated locally by ovarian factors. Initiation of follicular growth involves the transformation of primordial follicles from a quiescent, growtharrested state to a growth-committed state. This process is characterized by differentiation and proliferation of granulosa cells and by enlargement of the oocyte (Hirshfield 1991, Braw-Tel \& Yossefi 1997), and is regulated by several growth factors (Gougeon 1996, McGee \& Hsueh 2000, Picton \& Gosden 2000). Of intraovarian growth factors, attention has been focused on members of the TGF $\beta$ superfamily (Erickson \& Shimasaki 2000). Of these, growth differentiation factor-9 (GDF-9) is a growth factor secreted by the oocyte (Erickson \& Shimasaki 2000). GDF-9 mRNA and GDF-9 protein are not only expressed at the primary follicle stage but are also present in oocytes throughout growth to the ovulatory phase (Elvin et al.
1999, 2000, McGrath et al. 1995). In GDF-9 knockout mice (Dong et al. 1996), the absence of GDF-9 resulted in a block in folliculogenesis at the type 3b stage (late, onelayer primary follicle stage), indicating that GDF-9 is associated with early folliculogenesis.

The expression of GDF-9 mRNA and protein was confined to oocytes of primary and large follicles in rats (Hayashi et al. 1999, Jaatinen et al. 1999), mice (McGrath et al. 1995, Dong et al. 1996) and humans (Aaltonen et al. 1999). On the other hand, in sheep and cattle, GDF-9 mRNA was found in primordial follicles as well (Bodensteiner et al. 1999). These findings suggest the possibility that GDF-9 may be involved in the start of early folliculogenesis in a manner that differs among mammalian species. So far, it is unknown how GDF-9 is involved in early folliculogenesis in the porcine ovary.

Current ovarian stimulatory protocols for infertility treatment influence preantral and antral follicle growth mainly by using gonadotropins (Diedrich \& Felberbaum 
1998). However, a subset of patients has been found to be poor responders to gonadotropin stimulation (Scott 1996). For poor responders to gonadotropins, GDF-9 treatment may represent an alternative approach because of effects on primary and secondary follicle development. Indeed, recent studies showed that in vivo treatment with recombinant GDF-9 protein enhances ovarian weight and primordial as well as primary follicle progression up to the small preantral stage in neonatal rats (Hayashi et al. 1999). Our current study used an alternative method involving direct ovarian injection of gene fragments (Shimizu et al. 2003): direct ovarian injection of vascular endothelial growth factor gene fragments promotes follicular development in miniature gilts. In the present study, using our protocol for injection of gene fragments, we investigated the involvement of GDF-9 in early folliculogenesis in pigs. Since porcine GDF-9 cDNA has not been cloned, we first cloned the cDNA of porcine GDF-9 and examined how the direct ovarian injection of the cloned GDF-9 gene affected early folliculogenesis.

\section{Materials and Methods \\ Isolation of porcine cumulus-oocyte complexes (COCs)}

Porcine ovaries were obtained from prepubertal gilts at a slaughterhouse and carried to the laboratory within $30 \mathrm{~min}$ in a container kept at $37^{\circ} \mathrm{C}$. Follicles $2-5 \mathrm{~mm}$ in diameter in the ovaries were aspirated with a $5 \mathrm{ml}$ syringe with a 20-G needle, and only the COCs were collected. The COCs collected were then frozen and stored at $-80{ }^{\circ} \mathrm{C}$ until analysis.

\section{RT-PCR cloning of porcine GDF-9 cDNA}

Total RNA was isolated from the COCs with an RNeasy Mini Kit (Qiagen). Complementary DNA (cDNA) was synthesized with Ready-To-Go RT-PCR Beads (Amersham). The cDNA synthesized was used as the template in the polymerase chain reaction (PCR) with two degenerate primers, forward primer (5'-AAA GAC CAG CTG CAG CAT CC-3 $3^{\prime}$ ) and reverse primer (5'-TGG TGT GAA CTG GAG AGC CA-3'). The two primers were designed with reference to homologous sequences of bovine and ovine GDF-9 cDNA. The PCR was performed for 40 cycles at the annealing temperature of $55^{\circ} \mathrm{C}$. The resultant PCR fragment was cloned into pGEM-T Easy Vector (Promega), and five independent clones were sequenced.

The completed cDNA sequence of porcine GDF-9 was obtained by the rapid amplification of cDNA ends (RACE) technique. The sequence information of the PCR fragment was used to design primers for both $5^{\prime}$ RACE $\left(5^{\prime}\right.$-TGA CTC TTC TGG CAG CCC CCT CAC T-3') and $3^{\prime}$ RACE (5'-GGC CAC AAT CCA GTT GTC CCA CTT C-3'). RACE was performed with Advantage 2 Polymerase Mix by the procedure of the SMART RACE cDNA Amplification Kit (Clontech, Palo Alto, CA, USA). Both 5'RACE and $3^{\prime}$ RACE products were cloned into pGEM-T Easy Vector. The cloned fragments were sequenced by an ABI PRISM 310 DNA sequencer (Perkin Elmer, PE Applied Biosystems, Chiba, Japan).

\section{Animals and preparation of GDF-9 gene fragments for in vivo injection}

With the TAP Express Kit (Gene Therapy Systems, San Diego, CA, USA), porcine GDF-9 gene fragments were transformed into transcriptionally active PCR fragments for direct introduction into ovaries, according to the manufacturer's instructions. Briefly, custom oligos were used to produce the TAP primary fragment, which was then added to TAP Express promoter and terminator mixes. The $5^{\prime}$-custom and 3 '-custom oligos contained 44 and 43 nucleotides, respectively: of these, 26 (CTG CAG GCA CCG TCG TCG ACT TAA CA) and 25 (CAT CAA TGT ATC TTA TCA TGT CTG A) nucleotides were the 5'- and 3'-TAP Ends respectively. Universal sequence and the other 18 (GDF-9 sequence; 5'-ATG GCG CTT CCC AGA AAA-3' and 5'TTA ACG ACA CGT GCA CTT-3') nucleotides were used to make up the porcine GDF-9 sequences. In a preliminary examination, we confirmed that the green fluorescent protein (GFP) gene made with the kit was expressed in porcine fibroblasts. To investigate the effect of GDF-9 gene fragment injection on early folliculogenesis, seven 2month-old prepubertal gilts, mainly of the Large White breed, with a body weight of $20-24 \mathrm{~kg}$, were used, divided into two groups. After anesthetization by injection of ketamine hydrochloride ( $5 \mathrm{ml} /$ gilt; Sankyo Co., Tokyo, Japan) and atropine sodium salt $(0.5 \mathrm{mg} / \mathrm{gilt}$; Tanabe Co, Tokyo, Japan), one group $(n=3)$ that served as a control was directly injected with saline into the medulla of both ovaries with a 1-ml syringe with a 30-G needle. The other group ( $n=4$ ) was directly injected with GDF-9 gene fragments (10 $\mu \mathrm{g}$ DNA/ovary) into the medulla of both ovaries. GDF-9 gene fragments $(10 \mu \mathrm{g})$ were mixed with $10 \mu \mathrm{l}$ of GenePORTER reagent. Seven days after treatment, the ovaries in both groups were collected to examine the follicular population (the right ovary), and to detect mRNA expression (the left ovary). The present study was approved by the Ethics Committee for Care and Use of Laboratory Animals for Biomedical Research of the Graduate School of Agricultural Science, Tohoku University.

\section{Histological examination}

The right ovaries were fixed in $4 \%$ paraformaldehyde solution, embedded in paraffin wax and sectioned serially at a thickness of $6 \mu \mathrm{m}$. All sections were mounted on glass slides and stained with hematoxylin-eosin. The follicles were divided into one of the following four classes of the classification by Mossman and Duke (1973): (i) primordial follicles with a single layer of flattened pregranulosa cells; (ii) primary follicles with a single layer of cuboidal granulosa cells; (iii) secondary follicles with a stratified layer of cuboidal granulosa cells; (iv) tertiary follicles with 
multilayered granulosa cells. The follicles were counted and classified, using the oocyte nucleus as a marker to avoid several counts of the same oocyte. The percentage of primordial, primary, secondary and tertiary follicles in the ovarian cross-section was calculated on the basis of all follicles observed on randomly five sections having a medulla for each ovary.

\section{Quantitative real-time PCR}

PCR amplification was undertaken using the LightCycler System (Roche Diagnostics K.K., Tokyo, Japan). Total RNA of each porcine ovary was extracted as described above. Using SuperScript II (Invitrogen Japan K.K., Tokyo, Japan) and random primer in final volume of $20 \mu \mathrm{l}$, cDNA was synthesized from $0.5 \mu \mathrm{g}$ of RNA. To amplify the cDNA, $5 \mu \mathrm{l}$ aliquots of reverse-transcribed cDNA (diluted 1:5) were amplified by PCR in $20 \mu \mathrm{l}$ containing a final concentration of $3 \mathrm{mM} \mathrm{MgCl} 2,0.5 \mu \mathrm{M}$ of each primer (5'-TTC CCC AAA ACG AGT GTG AA-3'; 5'-AGT AGC GAG GGT TGT ATT TGT GTG-3') and 10\% LightCycler DNA master SRBR Green I (Roche Diagnostics K.K.). The reaction conditions were as follows: initial denaturation at $95^{\circ} \mathrm{C}$ for $10 \mathrm{~min}$ followed by 45 cycles of denaturation at $95^{\circ} \mathrm{C}$ for $10 \mathrm{~s}$, annealing at $56^{\circ} \mathrm{C}$ for $10 \mathrm{~s}$, and extension at $72^{\circ} \mathrm{C}$ for $20 \mathrm{~s}$ with a single fluorescence detection point at the end of the relevant extension segment. After this, one cycle of melting curve from $65^{\circ} \mathrm{C}$ to $97^{\circ} \mathrm{C}$, by a transition rate of $0.1{ }^{\circ} \mathrm{C} / \mathrm{s}$ with continuous detection of fluorescence, was performed. The temperature transition rate for all amplifications was $20^{\circ} \mathrm{C} / \mathrm{s}$. To control for the recovery of intact RNA and for the uniform efficiency of each reverse transcription reaction, a glyceraldehyde dehydrogenase $(\mathrm{GAPDH})$ fragment was amplified by real-time PCR using these primers: 5'-GAT GGT GAA GGT CGG AGT G-3' and 5'-CGA AGT TGT CAT GGA TGA CC-3'.

Quantitative analysis of the LightCycler data was performed by LightCycler software. The SYBR Green I signal of each sample was plotted against the number of cycles. This fluorescence threshold was used to determine the cycle number that correlated inversely with the log of the initial template concentration. Copy number analysis was undertaken by the 'second derivative maximum' mode and proportional baseline adjustment. The crossing points determined for GDF-9 mRNA were normalized to those of $\mathrm{GAPDH}$ to compensate for variability in RNA amount.

\section{Statistical analysis}

All data are presented as mean \pm S.E. The percentages of follicles at each different developmental stage were analyzed by the chi-square test. The significance of differences in the expression of genes was analyzed by Student's $t$-test. Differences were considered significant at $P<0.05$.

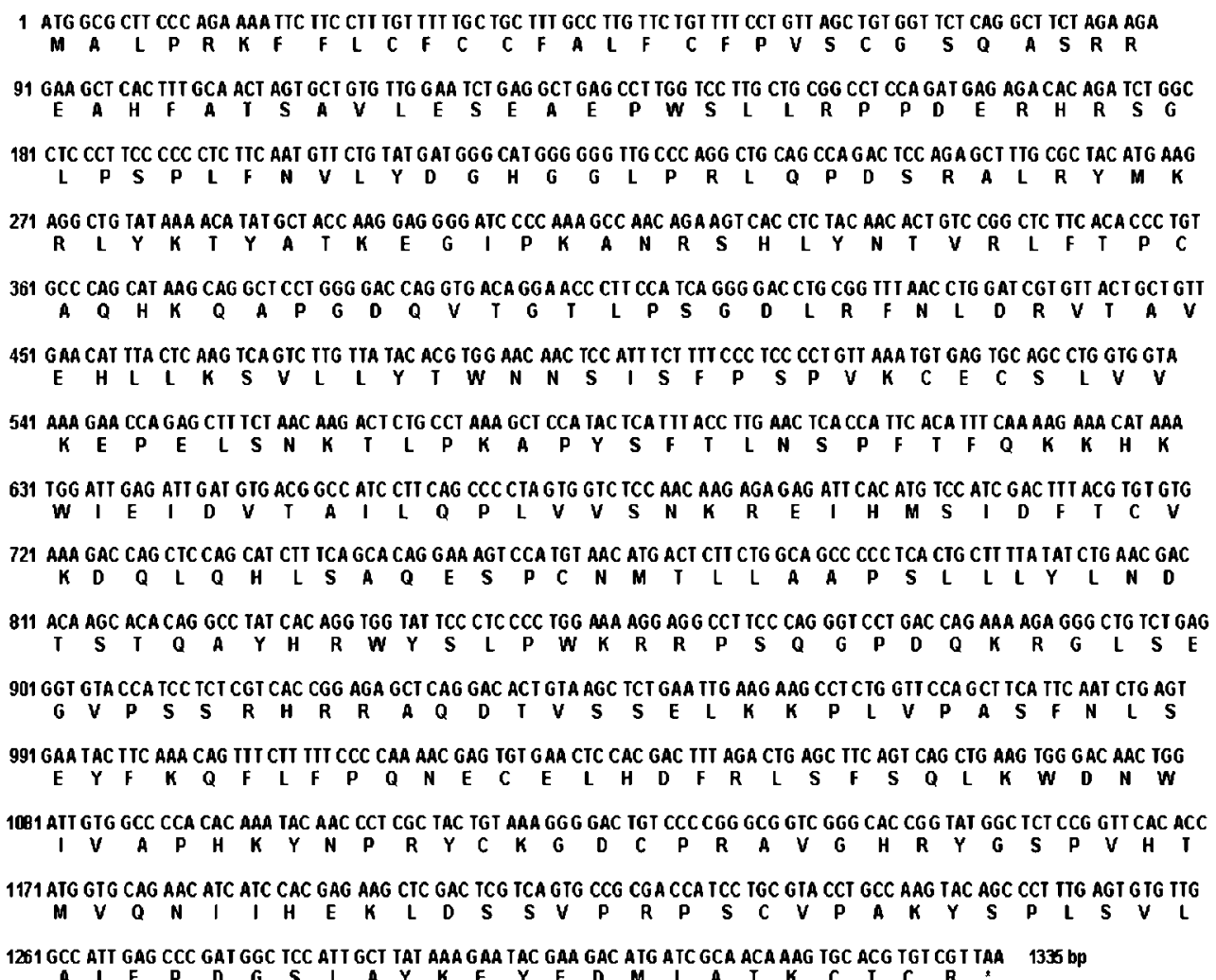

Figure 1 Nucleotide and deduced amino-acid sequence of the porcine GDF-9 cDNA. The mature protein sequence is numbered $1-445$. 
Results

Complementary DNA sequence of porcine GDF-9

Based on the nucleotide sequences of bovine and ovine GDF-9 cDNAs, we amplified GDF-9 cDNA from a porcine ovarian CDNA library and deduced the amino acid encoded thereby (Fig. 1). The open reading frame (ORF) of porcine GDF-9 cDNA is $1335 \mathrm{bp}$ long (encoding 445 amino acids, including the stop codon). Porcine GDF-9
Table 1 Homology of porcine GDF-9 ORF.

\begin{tabular}{lcc}
\hline Species & $\begin{array}{c}\text { cDNA } \\
\text { sequence }(\%)\end{array}$ & $\begin{array}{c}\text { Amino-acid } \\
\text { sequence }(\%)\end{array}$ \\
\hline Human & 81.4 & 76.9 \\
Mouse & 72.6 & 67.7 \\
Rat & 72.7 & 67.7 \\
Ovine & 84.2 & 79.7 \\
Bovine & 84.6 & 81.0 \\
\hline
\end{tabular}

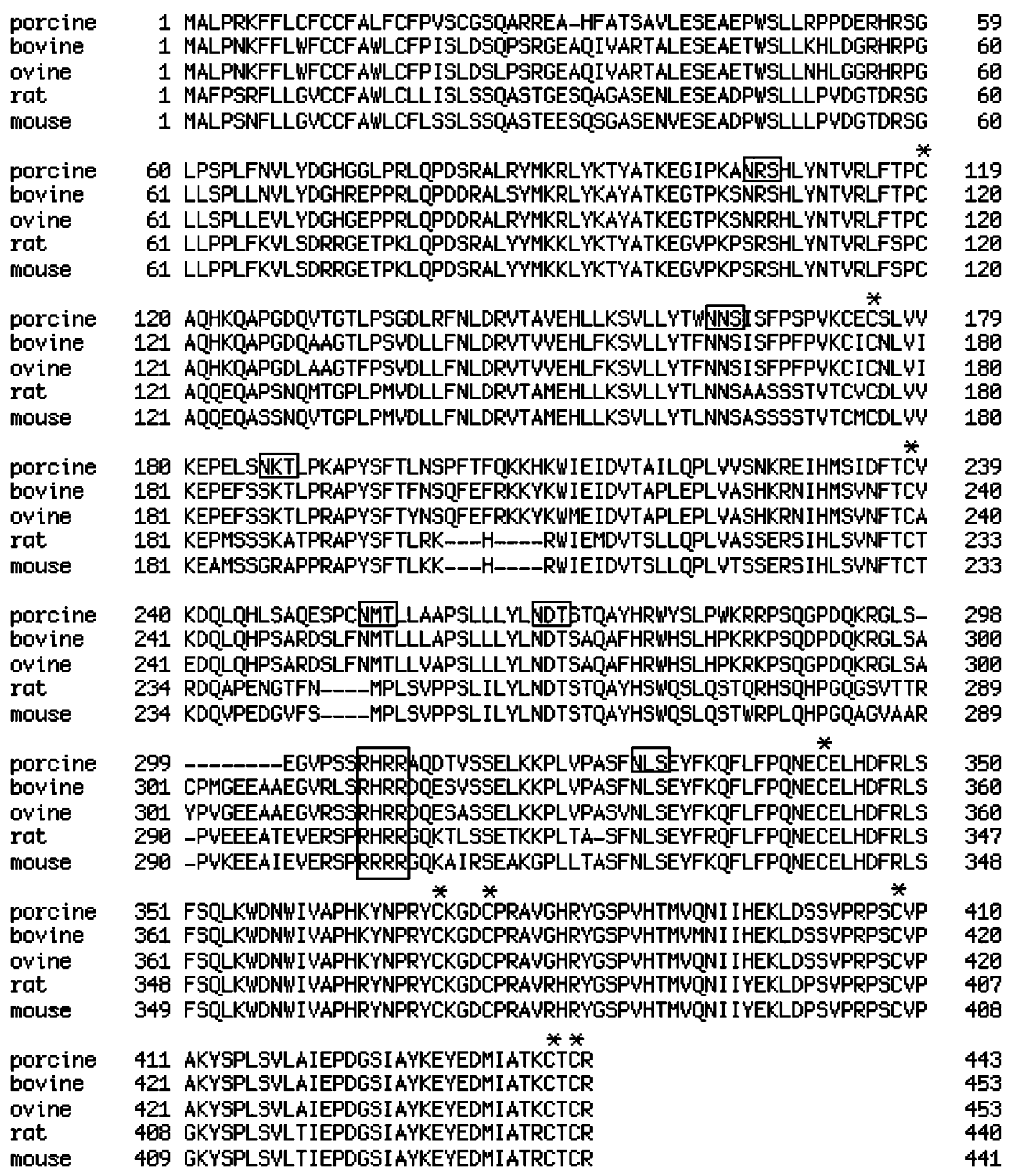

Figure 2 The characteristics of the deduced protein of porcine GDF-9. The deduced amino-acid sequence for porcine GDF-9 was compared with those of its bovine, ovine, rat and mouse counterparts. Porcine GDF-9 contains six potential N-glycosylation sites at asparagines 105 (NRS), 162 (NNS), 186 (NKT), 254 (NMT), 268 (NDT) and 327 (NLS), and a putative tetrabasic proteolytic processing site (RHRR) at amino acids 305-308. Asterisks represent conserved cysteine residues. 
has ORF homologies of $81.4 \%, 84.6 \%, 84.2 \%, 72.7 \%$ and $72.6 \%$ with its human (GenBank accession no. AC004500), bovine (GenBank accession no. AB058416), ovine (GenBank accession no. AF078545), rat (GenBank accession no. X81899) and mouse (GenBank accession no. BC052667) counterparts respectively (Table 1). For the putative mature protein, the corresponding homologies reach 92\%, 98\%, 97\%, 90\% and 88\% respectively. Porcine GDF-9 contains six potential $\mathrm{N}$-glycosylation sites at asparagines 105, 162, 186, 254, 268 and 327, and a putative tetrabasic proteolytic processing site (RHRR) at amino acids 305-308 (Fig. 2). In addition, multiple cysteines, presumably important for forming the disulfide bonds to maintain the tertiary structure of GDF-9, are also conserved.

\section{Folliculogenesis and expression of GDF-9 mRNA after GDF-9 gene fragments injection}

After injection of GDF-9 gene fragments, the percentage of primordial follicles in the ovaries decreased significantly compared with that in the control (Figs. 3 and 4).

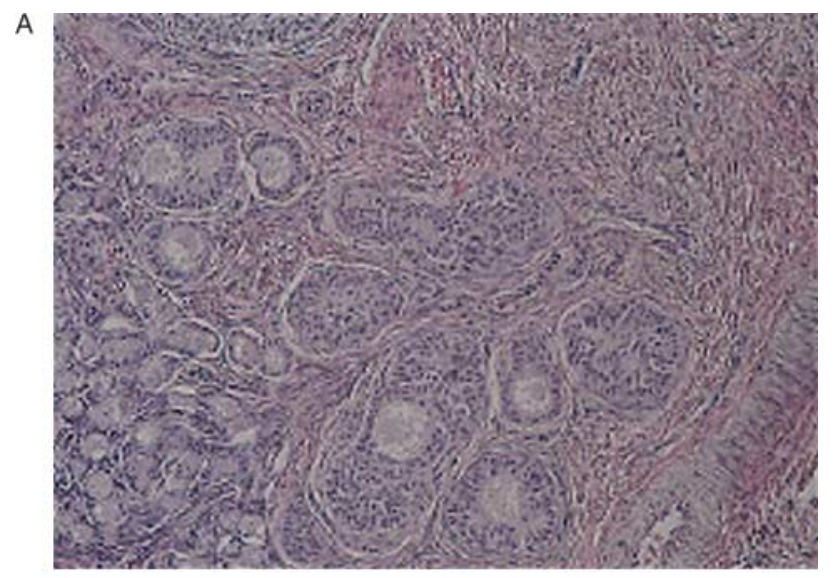

B

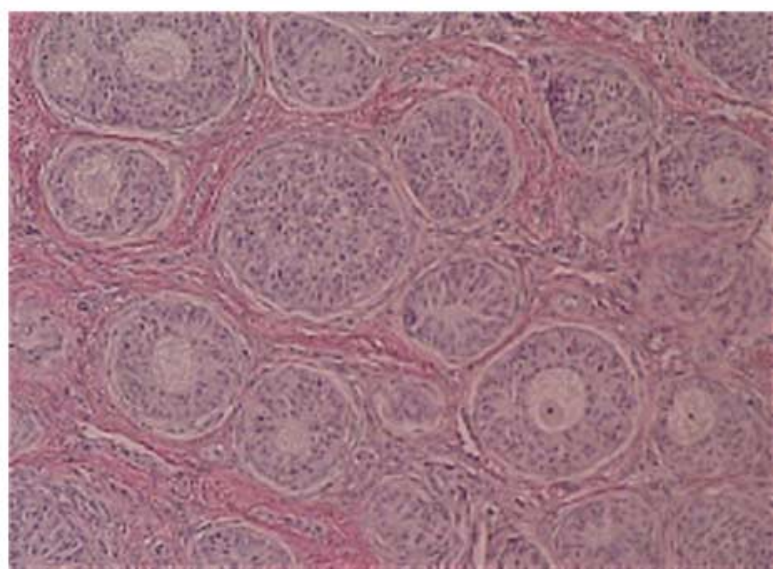

Figure 3 Micrographs of control (A) and porcine GDF-9 gene fragment-injected (B) ovaries of gilts. In porcine GDF-9 gene fragment-injected ovaries, mostly secondary and tertiary follicles emerged. All sections were stained with hematoxylin-eosin. Magnification $\times 200$. $\square$ Control $\square$ GDF-9

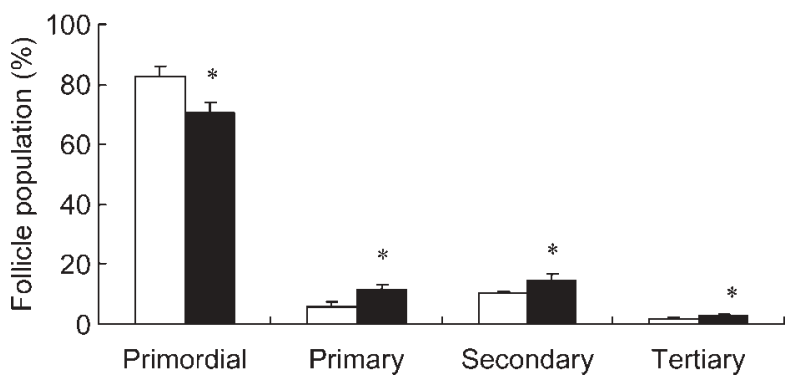

Figure 4 Changes of folliculogenesis in the ovaries injected with or without porcine GDF-9 gene fragments. The percentages of primary, secondary and tertiary follicles in the ovaries injected with GDF-9 gene fragments were significantly higher $(P<0.05$, chi-square test) than those in the control, whereas the percentage of primordial follicles in the ovary decreased significantly compared with that in the control.

The percentages of primary, secondary and tertiary follicles in the ovaries injected with GDF-9 gene fragments were significantly higher than those in the control (Fig. 4). Quantitative real-time PCR analysis showed that GDF-9 mRNAs were more intensively expressed in the GDF-9 gene fragment-treated ovaries than in the controls (Fig. 5).

\section{Discussion}

The GDF-9 ORF is composed of pro- and mature regions separated by a tetrabasic cleavage site (RHRR). Comparison of the amino-acid sequence of porcine GDF-9 with those of its bovine, ovine, rat and mouse counterparts indicated that the putative mature GDF-9 protein is highly conserved $(>88 \%)$ among these species. Multiple cysteines are conserved in all five species, and presumably are essential for the formation of disulfide bonds to stabilize the unique 'hand-shaped' protein (Daopin et al. 1992). In addition, one and five $\mathrm{N}$-linked glycosylation

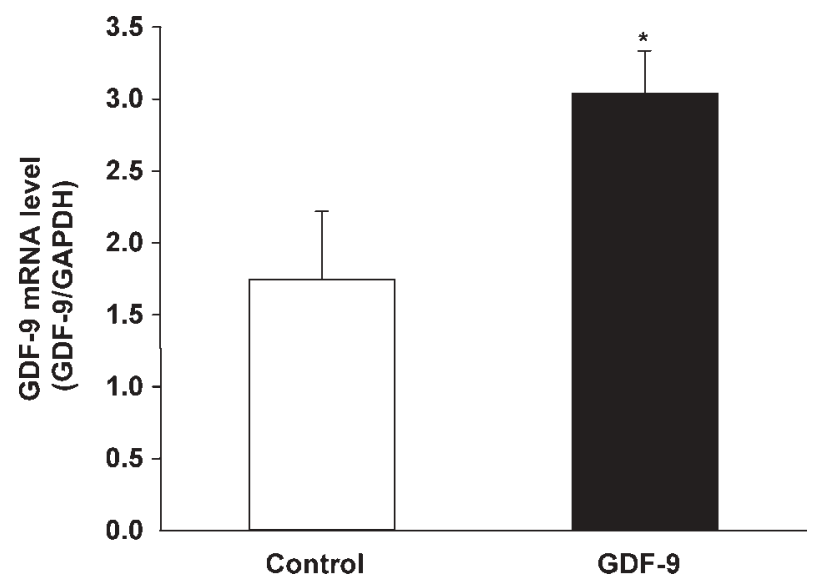

Figure 5 Expression of GDF-9 mRNA in porcine ovaries with or without injection of its gene fragment. Relative GDF-9 mRNA levels evaluated by real-time PCR and normalized to GAPDH mRNA levels. Asterisk indicates significant difference $(P<0.05)$. Data represent mean \pm S.E. 
sites are found in the mature and pro-region of porcine GDF-9 respectively. The porcine GDF-9 cDNA sequence reported in this study has been deposited in the DDBJ/GenBank/EMBL databases with accession no. AY649763.

Data from several studies suggest that GDF-9 is an important regulator of early follicle development. The GDF-9 mRNA transcript and protein are expressed in the oocytes of primary, but not primordial, follicles in mice (McGrath et al. 1995, Dong et al. 1996), rats (Hayashi et al. 1999, Jaatinen et al. 1999) and humans (Aaltonen et al. 1999). The presence of GDF-9 in primary and later stage follicles suggests that GDF-9-mediated signaling events may be important in the primary and later stages of follicle development. In contrast, GDF-9 mRNA is expressed in the primordial and subsequent stages of developing follicles in cattle and sheep (Bodensteiner et al. 1999). In the present study, to investigate the function of porcine GDF-9 in early folliculogenesis, we performed direct ovarian injection of porcine GDF-9 gene fragments by a technique previously developed (Shimizu et al. 2003). The results from the present study indicate that the injection of GDF-9 gene fragments increased the number of primary and secondary follicles, concomitant with a decrease in the number of primordial follicles. Our findings are consistent with previous findings that in vivo application of GDF-9 led to an increase in the number of primary and preantral follicles (Vitt et al. 2000a). Studies using GDF-9null mice indicated that follicles could grow to the primary stage (Dong et al. 1996), suggesting that GDF-9 may not necessarily be required for the transition from primordial to primary follicles. However, our findings showed that exogenous GDF-9 in the porcine ovary may accelerate the transition from primordial to primary follicles, supporting the previous finding (Vitt et al. 2000a) that once GDF-9 is produced by the oocyte of a given primordial follicle that follicle could begin to grow, although the origin of the GDF-9 present at the beginning of folliculogenesis is unknown. In addition, the present study demonstrated that the injection of GDF-9 gene fragments resulted in an increase in the number of secondary and tertiary follicles. Since GDF-9 promotes granulosa cell proliferation of early antral and preovulatory follicles but inhibits FSH-induced steroidogenesis and LH receptor expression (Vitt et al. 2000b), our findings suggest that GDF-9 may stimulate granulosa cell proliferation of secondary and tertiary follicles, by which the resultant growth of these follicles may be accelerated.

A previous study indicated that the level of GDF-9 mRNA appeared to decrease in both primary oocytes of polycystic ovary syndrome (PCOS) and polycystic ovaries (PCOs) during the growth and differentiation phases (Teixeira et al. 2002). These findings suggested that GDF-9 mRNA expression in the vast majority of PCOS/PCO oocytes did not begin until follicles had reached the primary/secondary transition stage. The use of direct ovarian injection of GDF-9 gene fragments applied in the present study may contribute to development of novel therapies for prevention and treatment of infertility associated with conditions of ovarian dysfunction such as PCOS and PCOs.

In conclusion, porcine GDF-9 was cloned here for the first time, and injection of its gene fragments into the ovary resulted in an increase in the number of primary, secondary and tertiary follicles. These results demonstrated the enhancement of the transition from the primordial to primary follicle stages. Current ovarian stimulation protocols for infertility treatment influence antral follicle growth mainly by using gonadotropins (Diedrich \& Felberbaum 1998). However, in poor responders to gonadotropin stimulation (Scott 1996), it is difficult to enhance the ovulation rate by current infertility therapy. Oocyte donation is the only option for such patients desiring pregnancy (Kalantaridou et al. 1998). Ovaries from most women with premature ovarian failure contain primordial follicles (Oliver 1995). Therefore, injection of GDF-9 gene fragments into the ovary may be an effective method to make primordial follicles grow. We believe that our study may offer an innovative protocol for the development of novel therapies for prevention and treatment of infertility associated with ovarian dysfunction.

\section{Acknowledgement}

This research was supported by the Program for Promotion of Basic Research Activities for Innovative Biosciences and by grants from Bio-orientated Technology Research Advancement Institution (BRAIN), Japan.

\section{References}

Aaltonen J, Laitinen MP, Vuojolainen K, Jaatinen R, Horelli-Kuitunen N, Seppa L, Louhio H, Tuuri T, Sjoberg J, Butzow R, Hovata $\mathbf{O}$, Dale L \& Ritvos O 1999 Human growth differentiation factor-9 (GDF-9) and its novel homolog GDF-9B are expressed in oocytes during early folliculogenesis. Journal of Clinical Endocrinology and Metabolism 84 2744-2750.

Bodensteiner KJ, Clay CM, Moeller CL \& Sawyer HR 1999 Molecular cloning of the ovine growth/differentiation factor-9 gene and expression of growth/differentiation factor-9 in ovine and bovine ovaries. Biology of Reproduction $60381-386$.

Braw-Tel R \& Yossefi S 1997 Studies in vivo and in vitro on the initiation of follicle growth in the bovine ovary. Journal of Reproduction and Fertility 109 165-171.

Daopin S, Piez KA, Ogawa Y \& Davies DR 1992 Crystal structure of transforming growth factor-beta 2: an unusual fold for the superfamily. Science 257 369-373.

Diedrich K \& Felberbaum R 1998 New approaches to ovarian stimulation. Human Reproduction 13 (Suppl) 1-13.

Dong J, Albertini DF, Nishimori K, Kumar TR, Lu N \& Matzuk MM 1996 Growth differentiation factor-9 is required during early ovarian folliculogenesis. Nature 383 531-535.

Elvin JA, Clark AT, Wang P, Wolfman NM \& Matzuk MM 1999 Paracrine actions of growth differentiation factor-9 in the mammalian ovary. Molecular Endocrinology 13 1035-1048.

Elvin JA, Yan C \& Matzuk MM 2000 Oocyte-expressed TGF- $\beta$ superfamily members in female fertility. Molecular and Cellular Endocrinology 159 1-5.

Erickson GF \& Shimasaki S 2000 The role of the oocyte in folliculogenesis. Trends in Endocrinology and Metabolism 11 193-198. 
Gougeon A 1996 Regulation of ovarian follicular development in primate: facts and hypothesis. Endocrine Reviews 17 121-155.

Hayashi M, McGee EA, Min G, Klein C, Rose UM, van Duin M \& Hsueh AJ 1999 Recombinant growth differentiation factor-9 (GDF-9) enhances growth and differentiation of cultured early ovarian follicles. Endocrinology 140 1236-1244.

Hirshfield AN 1991 Development of follicles in the mammalian ovary. International Review of Cytology 124 43-101.

Jaatinen R, Laitinen MP, Vuojolainen K, Aaltonen J, Louhio $\mathbf{H}$ Heikinheimo K, Lehtonen E \& Ritvos O 1999 Localization of growth differentiation factor-9 (GDF-9) mRNA and protein in rat ovaries and cDNA cloning of rat GDF-9 and its novel homolog GDF-9B. Molecular and Cellular Endocrinology 156 189-193.

Kalantaridou SN, Davis SR \& Nelson LM 1998 Premature ovarian failure. Endocrinology and Metabolism Clinics of North America 27 989-1006.

McGee EA \& Hsueh AJ 2000 Initial and cyclic recruitment of ovarian follicles. Endocrine Review 21 200-214.

McGrath SA, Esquela AF \& Lee SJ 1995 Oocyte-specific expression of growth/differentiation factor-9. Molecular Endocrinology 9 $131-136$.

Mossman HW \& Duke KL 1973 Comparative Morphology of the Mammalian Ovary. Madison, WI: University of Wisconsin Press.

Oliver AC 1995 The role of laparoscopic ovarian biopsy in the management of premature gonadal failure. Journal of the American Association of Gynecologic Laparoscopists 2 S37.

Picton HM \& Gosden RG 2000 In vitro growth of human primordial follicles from frozen-banked ovarian tissues. Molecular and Cellular Endocrinology 166 27-35.
Scott RT 1996 Evaluation and treatment of low responders. Seminars in Reproductive Endocrinology 14 317-337.

Shimizu T, Jiang JY, lijima K, Miyabayashi K, Ogawa Y, Sasada H \& Sato E 2003 Induction of follicular development by direct single injection of vascular endothelial growth factor (VEGF) gene fragments into the ovary of miniature gilts. Biology of Reproduction $\mathbf{6 9}$ $1388-1393$

Teixeira Filho FL, Baracat EC, Lee TH, Suh CS, Matsui M, Chang RJ, Shimasaki S \& Erickson GF 2002 Aberrant expression of growth differentiation factor-9 in oocytes of women with polycystic ovary syndrome. Journal of Clinical Endocrinology and Metabolism 87 $1337-1344$

Vitt UA, McGee EA, Hayashi M \& Hsueh AJW 2000a In vivo treatment with GDF-9 stimulates primordial and primary follicle progression and theca cell marker CYP17 in ovaries of immature rats. Endocrinology 141 3814-3820.

Vitt UA, Hayashi M, Klein C \& Hsueh AJW 2000b Growth differentiation factor-9 stimulates proliferation but suppresses the folliclestimulating hormone-induced differentiation of cultured granulosa cells from small antral and preovulatory rat follicles. Biology of Reproduction 62 370-377.

Received 19 March 2004

First decision 27 April 2004

Revised manuscript received 21 June 2004

Accepted 16 July 2004 